\title{
How good are we at modeling implantation?
}

\section{Aghajanova Lusine $^{1}$}

Received: 9 December 2019 / Accepted: 12 December 2019/Published online: 20 December 2019

(C) Springer Science+Business Media, LLC, part of Springer Nature 2019

Keywords Implantation $\cdot$ Endometrium $\cdot$ Embryo $\cdot$ Implantation model $\cdot$ Co-culture

With the development of IVF, some of the mystery around human reproduction has unfolded by being successfully replicated in a dish (or "tube"). This correlates to processes such as fertilization of egg by sperm, embryo development, cleavage, and blastulation. However, one of the key processes in human reproduction, such as implantation, remains to be a "black box" and a major limitation of the IVF success [1]. We believe that clarifying the details involved in the human implantation process will help to solve many cases of infertility and early pregnancy loss.

The far-reaching goals of reproductive biologists in regard to the uterus are ranging from improving implantation of transferred embryos to completely outsourcing the pregnancy by developing an artificial uterus. This will need to not only involve a successful implantation of human embryo into an artificially created endometrium, but also to be followed by invasion and placentation, and successful support of pregnancy up to term. Peeking into the future, the successful ectogenesis (the implantation and full embryo/fetal development in vitro) will not only solve the problems around implantation failure and possibly pregnancy loss but will also tackle the mega-issue of preterm birth.

So, where are we on this long bumpy road? Studying human implantation process is challenging and tricky. The methods in which research had been conducted up to recently involved heavy utilization of endometrial biopsies from patients and healthy volunteers, and super numeral donated human embryos, and studying them separately. This approach, however, presents an in vivo snapshot of complex interactions between embryo and endometrium rather than a dynamic

Aghajanova Lusine

aghajano@stanford.edu

1 Division of Reproductive Endocrinology and Infertility, Department of Obstetrics and Gynecology, Stanford University School of Medicine, 1195 West Fremont Avenue, MC 7717,

Sunnyvale, CA 94087, USA system. Animal models provide a valuable insight into what could be an important target to study. However, it is common knowledge that the data cannot be directly extrapolated from animal to human due to many dissimilarities, such as difference in decidualization (in mice, it happens in the presence of an embryo only), LIF knock-out mice are infertile, while this has not been proven in human, and many others. Nevertheless, while animal studies provide an extremely valuable source of knowledge and the need to perform respective human studies is apparent, the major limitation for research in human is still the availability of human cells/tissue (and several others) [2]. Human decidual tissue from pregnancy terminations or ectopic pregnancy has its own limitations and clearly does not represent the "non-pregnant endometrium" prior to implantation.

One of the valuable ways of studying human implantation is creating in vitro models of embryo-endometrial interaction that mimic the in vivo situation as close as possible. The initial approach was to obtain the needed players and put them together in a co-culture system, such as utilization of endometrial tissue explants, with its 3-D structure and all the layers of endometrium preserved. The embryo was then being placed on endometrial lining, with subsequent observation of the penetration of the lining by a blastocyst [3]. Before that, Kliman et al. [4] placed minced endometrial tissue with trophoblast cells into an in vitro suspension system, and they were able to demonstrate attachment and even invasion of trophoblast cells. Explant culture had its own limitations, and most of the subsequent studies were utilizing isolated endometrial epithelial and/or stromal cells, and human preimplantation embryos (blastocysts) as monolayer [5] or multilayer co-cultures [6, 7]. For the latter, in a 3-D co-culture system, endometrial stromal cells were embedded in a collagen matrix and separated from the endometrial epithelial cell monolayer by basement membrane material-Matrigel. The cultures were then treated with estrogen and progesterone mimicking the secretory phase of the menstrual cycle and aiming at stimulating the receptive endometrium, with subsequent placement of an embryo on top of epithelium [8]. 
However, there are obvious challenges in obtaining human embryos for research. Therefore, with increasing frequency, the human embryos are being replaced by trophoblast tissue from early pregnancy terminations [9]. Moreover, governmental restrictions on human embryo use has lead to substitution of human embryos in co-culture system models by human embryonic stem cell (hESC)-derived trophoblast cells and human-induced pluripotent stem cells (hiPSC).

Advances in imaging techniques such as live imaging of cells and embryo development [10] lead to our better understanding of specific spatial and temporal relationship between cells: the endometrium and the implanting embryo. The "indirect" model of implantation was recently reported by Zheng et al. [11] in a microfluidic device that beautifully modeled the early events in human embryo development upon implantation by using hESC and hiPSC. The endometrial participation was mimicked by the supplementing basal media with specific growth factors in appropriate channels. This nicely built on previous achievements by the same group and development of iPSC [12].

This issue of JARG features an elegant study by Evans and colleagues (a novel "embryo-endometrial" adhesion model can potentially predict "receptive" or "non-receptive" endometrium; https://doi.org/10.1007/s10815-019-01629) describing the utilization of trophectoderm cells derived from hESC developed from individual blastomeres of donated human embryos. The group established a model of initial stages of human implantation using trophectodermal spheroids formed from these trophoblast stem cells and various human endometrial epithelial cell lines (primary cells from fertile and infertile women, Ishikawa endometrial adenocarcinoma cells, ECC-1 endometrial cancer cell line, and two endometrial epithelial cells lines). The endometrial epithelial cells were pre-treated with estrogen and progesterone prior to adding the spheroids, and the attachment and outgrowth of the spheroids was determined. The results showed that trophectodermal spheroid attachment was hormonally dependent and was significantly higher in fertile women. The latter observation is quite remarkable, as it shows that this trophectoderm spheroid attachment model can discriminate between endometrial epithelial cells from fertile versus infertile women with unexplained (presumably uterine factor) infertility, based on adhesion properties. Interestingly, the notion of endometrial cells selecting an embryo has been suggested before [13].

Thus, it is logical to speculate that this new model of embryo-endometrial implantation could be utilized as an in vitro "diagnostic" tool of endometrial infertility. While this is still far from clinical use as a potential diagnostic tool of "implantable vs unimplantable" endometrium (or embryo?), it is a path worth pursuing in the future.

So, how good are we at this point at modeling human implantation? While we still lack robust, highly reproducible, and readily assembled models, substantial progress has been made. No one will argue that using efficient models of human implantation will enable easier research to understand the intricacies of interactions between endometrium and trophectoderm cells, and offer a venue for testing possible therapeutic interventions. Reliable and tractable models will allow substitution of endometrial and embryonic components of the model with cells from patients with different uterine conditions such as recurrent pregnancy loss, implantation failure, endometriosis, and adenomyosis. Moreover, the development and availability of human iPSC lines and blastocyst-like entities from healthy patients or those bearing various disease conditions makes for a promising reconstitution paradigm with which mechanistic studies on implantation could proceed.

To summarize, with many questions still unanswered, we clearly need robust models of human implantation that can reflect the real-life conditions as close as possible for increasing our success in battling fertility issues and to initiate desired (for some) future with ectogenesis.

\section{References}

1. Macklon NS, Geraedts JP, Fauser BC. Conception to ongoing pregnancy: the 'black box' of early pregnancy loss. Hum Reprod Update 2002;8(4):333-343. Epub 2002/09/11. doi: https://doi.org/ 10.1093/humupd/8.4.333.

2. Teklenburg G, Macklon NS. Review: in vitro models for the study of early human embryo-endometrium interactions. Reprod Sci. 2009;16(9):811-8. Epub 2009/04/28. https://doi.org/10.1177/ 1933719109334966.

3. Landgren BM, Johannisson E, Stavreus-Evers A, Hamberger L, Eriksson H. A new method to study the process of implantation of a human blastocyst in vitro. Fertil Steril. 1996;65(5):1067-70. Epub 1996/05/01. https://doi.org/10.1016/s0015-0282(16)582910 .

4. Kliman HJ, Feinberg RF, Haimowitz JE. Human trophoblastendometrial interactions in an in vitro suspension culture system. Placenta. 1990;11(4):349-367. Epub 1990/07/01. doi: https://doi. org/10.1016/s0143-4004(05)80226-7.

5. Galan A, O'Connor JE, Valbuena D, Herrer R, Remohi J, Pampfer $\mathrm{S}$, et al. The human blastocyst regulates endometrial epithelial apoptosis in embryonic adhesion. Biol Reprod. 2000;63(2):430-9.

6. Bentin-Ley U, Pedersen B, Lindenberg S, Larsen JF, Hamberger L, Horn T. Isolation and culture of human endometrial cells in a threedimensional culture system. J Reprod Fertil. 1994;101(2):327-32.

7. Boggavarapu NR, Berger C, von Grothusen C, Menezes J, Gemzell-Danielsson K, Lalitkumar PG. Effects of low doses of mifepristone on human embryo implantation process in a threedimensional human endometrial in vitro co-culture system. Contraception. 2016;94(2):143-51. Epub 2016/03/24. https://doi. org/10.1016/j.contraception.2016.03.009.

8. Berger C, Boggavarapu NR, Menezes J, Lalitkumar PG, GemzellDanielsson K. Effects of ulipristal acetate on human embryo attachment and endometrial cell gene expression in an in vitro co-culture system. Hum Reprod 2015;30(4):800-811. Epub 2015/03/06. doi: https://doi.org/10.1093/humrep/dev030.

9. Popovici RM, Betzler NK, Krause MS, Luo M, Jauckus J, Germeyer A, et al. Gene expression profiling of human 
endometrial-trophoblast interaction in a coculture model. Endocrinology. 2006;147(12):5662-75. Epub 2006/09/02. https:// doi.org/10.1210/en.2006-0916.

10. White MD, Zenker J, Bissiere S, Plachta N. Instructions for assembling the early mammalian embryo. Dev Cell 2018;45(6):667-679. Epub 2018/06/20. doi: https://doi.org/10.1016/j.devcel.2018.05. 013.

11. Zheng Y, Xue X, Shao Y, Wang S, Esfahani SN, Li Z, et al. Controlled modelling of human epiblast and amnion development using stem cells. Nature. 2019;573(7774):421-5. Epub 2019/09/13. https://doi.org/10.1038/s41586-019-1535-2.

12. Nakamura T, Okamoto I, Sasaki K, Yabuta Y, Iwatani C, Tsuchiya H, Seita Y, Nakamura S, Yamamoto T, Saitou M. A developmental coordinate of pluripotency among mice, monkeys and humans.
Nature. 2016;537(7618):57-62. Epub 2016/08/25. doi: https://doi. org/10.1038/nature19096.

13. Teklenburg G, Salker M, Molokhia M, Lavery S, Trew G, Aojanepong T, Mardon HJ, Lokugamage AU, Rai R, Landles C, Roelen BA, Quenby S, Kuijk EW, Kavelaars A, Heijnen CJ, Regan L, Brosens JJ, Macklon NS. Natural selection of human embryos: decidualizing endometrial stromal cells serve as sensors of embryo quality upon implantation. PLoS One. 2010;5(4):e10258. Epub 2010/04/28. doi: https://doi.org/10.1371/journal.pone.0010258. PubMed PMID: 20422011; PMCID: PMC2858159.

Publisher's note Springer Nature remains neutral with regard to jurisdictional claims in published maps and institutional affiliations. 